\title{
UTILIZAÇÃO DE SOLOS LOCAIS PARA TRATAMENTO DE EFLUENTES DO REFINO DE PETRÓLEO CONTENDO METAIS
}

Rafael Midugno*, Ari Roisenberg, Antonio Pedro Viero e Eduardo Sanberg

Departamento de Mineralogia e Petrologia, Instituto de Geociências, Universidade Federal do Rio Grande do Sul, CP 15001, 90650-000 Porto Alegre - RS, Brasil

Nicolai Mirlean

Departamento de Geociências, Fundação Universidade Federal do Rio Grande, CP 474, 96201-900 Rio Grande - RS, Brasil

Recebido em 18/7/06; aceito em 30/1/07; publicado na web em 30/7/07

\begin{abstract}
EMPLOYMENT OF LOCAL SOILS FOR TREATMENT OF METAL-BEARING WASTEWATER FROM PETROLEUM REFINEMENT. The employment of local soils for extraction of metallic elements was evaluated through batch tests to treat wastewaters generated in a petroleum refinery plant in southern Brazil. Clay and organic carbon content and clay mineralogy provide these soils, in principle, with moderate metal retention capacity. The following retention order was established: $\mathrm{Cr}^{3+}>\mathrm{Pb}^{2+}>\mathrm{Cu}^{2+}$ $>\mathrm{Hg}^{2+}>\mathrm{Cd}^{2+}$, with total amount of metals retained varying from 36 to $65 \mathrm{meq} \mathrm{kg}{ }^{-1}$. The results show the high efficiency of local soils for extracting metals from liquid effluents through sorption and precipitation processes under acid $\mathrm{pH}$ conditions.
\end{abstract}

Keywords: retention test; metal pollution; wastewater treatment.

\section{INTRODUÇÃO}

A contaminação de solos e águas superficiais e subterrâneas por atividades industriais tem tornado as áreas urbanizadas cenários de risco ambiental ${ }^{1}$. Em refinarias de petróleo, os efluentes apresentam altas concentrações de complexos metálicos, em especial, cromo, cobre, mercúrio, zinco e chumbo. A toxicidade desses elementos varia de acordo com temperatura, salinidade, $\mathrm{pH}$ e número de oxidação e, adicionalmente, podem apresentar comportamento sinérgico com outros elementos metálicos ${ }^{2}$, potencializando o impacto sobre a saúde da biota que compõe o meio ambiente.

Diversos materiais têm sido usados na remoção de contaminantes em águas residuais, dentre os quais, carbono ativado, organoargilas ${ }^{3}$, turfas $^{4}$ e zeólitas ${ }^{5}$ que são materiais de fácil obtenção e disposição ${ }^{6}$. Os solos de granulometria argilosa, constituídos por argilominerais e matéria orgânica, apresentam uma grande afinidade com íons metálicos e podem, dependendo das condições físico-químicas, reter ou liberar cátions ${ }^{7}$. Como resultado, esses solos podem atuar tanto como purificadores quanto como fontes potenciais de poluição.

A capacidade dos solos em reter elementos metálicos pode ser determinada através de testes de lixiviação ("column tests") e em série ("batch tests") ${ }^{8,9}$. Conceitualmente, os testes em série assumem um modelo físico de sistema disperso de partículas de solo, onde as superfícies estão completamente expostas e disponíveis para interações com os íons em solução. A Agência de Proteção Ambiental dos Estados Unidos (USEPA) possui um conjunto de técnicas normatizadas ${ }^{10} \mathrm{e}$ aplicadas correntemente na determinação da capacidade de retenção de produtos químicos por solos.

Na Refinaria Alberto Pasqualini, localizada em Canoas/RS, identificou-se área de disposição inadequada de resíduos ${ }^{11}$. Denominada "borreiro", esta área permaneceu ativa por cerca de 15 anos, recebendo tanto resíduos líquidos quanto sólidos sem tratamento. A prática da descarga de efluentes diretamente sobre a superfície do solo acarreta sérios riscos ambientais e à saúde das populações atingidas. No entanto, a avaliação do impacto ambiental na área da

*e-mail: rafael.midugno@ufrgs.br
Refinaria demonstrou que aterros e solos ocorrentes nesta área impediram a disseminação da carga poluente além dos limites de seu território $^{12}$.

Aproveitando que solos podem possuir boa capacidade de retenção e degradação de poluentes tóxicos ${ }^{13}$, unidades de tratamento de efluentes industriais vêm empregando esses materiais como filtros naturais de geoacumulação. Basílio et al. ${ }^{14}$ avaliaram o uso de sedimentos provenientes de barragens de contenção do processamento de minério de ferro localizadas no Quadrilátero Ferrífero, Minas Gerais, como alternativa de baixo custo para minimizar a contaminação de elementos metálicos em águas por processos de adsorção nas fases minerais dos sedimentos.

O objetivo do presente trabalho é analisar o comportamento e a potencialidade do emprego de solos locais como imobilizadores de poluentes ricos em íons metálicos $-\mathrm{Cr}, \mathrm{Cu}, \mathrm{Cd}, \mathrm{Hg}$ e $\mathrm{Pb}-$ a fim de constituírem unidades de tratamento de efluentes gerados na própria Refinaria.

\section{PARTE EXPERIMENTAL}

\section{Amostras de solo}

Foram selecionadas 3 amostras representativas dos níveis mais superficiais dos solos ocorrentes na área da Refinaria para a realização de ensaios de retenção do tipo em série. A profundidade de coleta das amostras (denominadas a, b e c) é, respectivamente, 0,5; 1,5 e 1,2 m. As frações granulométricas presentes, argila, silte e areia, estão distribuídas percentualmente e respectivamente nas amostras da seguinte forma: amostra a: 31,9; 18,9 e 49,2; amostra b: 60,$8 ; 37,0$ e 2,2 ; e amostra c: 54,$9 ; 4,5$ e 40,6.

Classificadas texturalmente segundo a razão argila-silte-areia de Shepard ${ }^{15}$, as amostras foram enquadradas como a: areia argilosa, b: argila siltosa e c: argila arenosa (Figura 1).

Análises por difratometria de Raios-X apontam ampla predominância de caolinita na fração argilosa, acompanhada de montmorilonita e quartzo e com quantidades subordinadas de muscovita e albita $^{11}$ 


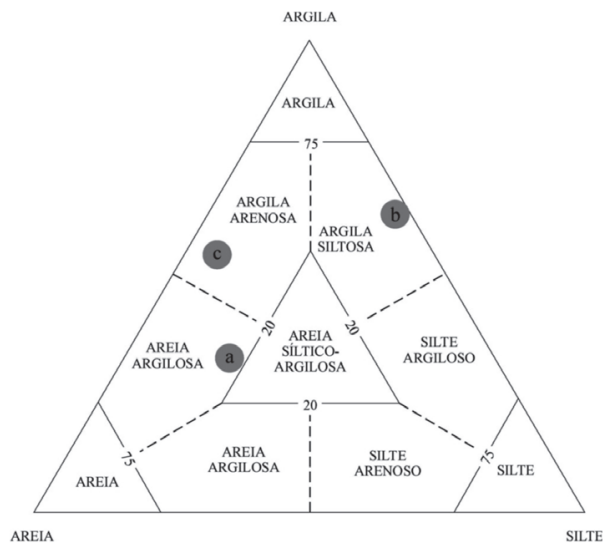

Figura 1. Classificação dos solos da REFAP de acordo com o diagrama de Shepard

\section{Preparação das soluções experimentais}

Cinco dos principais elementos metálicos presentes nos solos e águas subterrâneas da Refinaria, a saber, cromo, cobre, cádmio, mercúrio e chumbo, foram selecionados para os testes de retenção. Os elementos metálicos, obtidos sob a forma de sais solúveis $\mathrm{CrCl}_{3}$, $\mathrm{CuCl}_{2}, \mathrm{CdCl}_{2}, \mathrm{~Pb}\left(\mathrm{NO}_{3}\right)_{2}$ e $\mathrm{HgCl}_{2}$ foram diluídos em soluções contendo água deionizada na seguinte proporção: $1,0 \mathrm{mmol}$ de $\mathrm{Cr}, \mathrm{Cu}$, $\mathrm{Cd}$ e $\mathrm{Pb}$ e $0,5 \mathrm{mmol}$ de $\mathrm{Hg}$, perfazendo um conteúdo total de 4,5 mmol de íons metálicos.

As soluções preparadas em laboratório atingiram valores de pH mais homogêneos, entre 3,75 e 4,42, se comparadas às soluções coletadas em campo, que exibiram valores entre 5,10 e 8,00. Com isso, é esperada uma maior mobilidade dos íons metálicos em solução durante os testes de retenção.

\section{Procedimentos experimentais e analíticos}

A capacidade de retenção dos solos foi determinada através de uma bateria de testes em série com intervalo crescente de tempo de interação: 1, 3, 12, 24 e 48 h.

Inicialmente, os solos foram submetidos a um pré-processamento que compreende secagem das amostras em estufa a $60{ }^{\circ} \mathrm{C}$, durante $24 \mathrm{~h}$, desagregação do material com pistilo e gral, quarteamento e pesagem.

O procedimento de interação solução-solo foi realizado em 15 frascos fechados de polipropileno de $100 \mathrm{~mL}$ (um para cada tempo de interação), contendo a solução aquosa metal-catiônica e 2,0000 $\pm 0,0005 \mathrm{~g}$ de solo. A separação da carga sólida em cada tempo foi conduzida mediante filtragem com membranas Milipore 0,45 $\mu \mathrm{m}$, seguida de 20 min de centrifugação a $10.000 \mathrm{rpm}$ e $25^{\circ} \mathrm{C}$. A solução residual foi então encaminhada para análise em laboratório certificado pelo órgão ambiental estadual.

As concentrações de íons metálicos em solução foram medidas no espectrofotômetro de absorção atômica com forno de grafite, marca Perkin Elmer/modelo AAS Analyst 800. O laboratório utiliza os procedimentos analíticos contidos nos métodos EPA 200.7; 200.8 e 200.13. No caso especial do mercúrio, o método de análise emprega vapor a frio. A exatidão dos resultados analíticos foi calculada pela variação percentual entre um determinado intervalo amostral e o intervalo imediatamente anterior para um nível de probabilidade 0,05 .

\section{Determinação da capacidade de retenção de elementos metálicos}

A capacidade de retenção de elementos metálicos é uma medida útil na definição da aplicabilidade de solos locais como geoacumu- ladores, pois define a quantidade máxima de contaminantes que poderão ser removidos de solução.

A capacidade de retenção de elementos metálicos (R) é obtida através da seguinte expressão:

$\mathrm{R}=\left[\left(\mathrm{C}_{\mathrm{i}}-\mathrm{C}_{\mathrm{x}}\right) \cdot \mathrm{V}\right] \cdot \mathrm{W}^{-1}$

sendo: $\mathrm{C}_{\mathrm{i}}=$ concentração inicial de elementos metálicos na solução; $C_{x}=$ concentração de elementos metálicos na solução em determinado intervalo amostral; $\mathrm{V}=$ volume de solução; $\mathrm{W}=$ massa de amostra de solo.

\section{RESULTADOS E DISCUSSÃO}

Os resultados obtidos indicam queda na concentração de todos os elementos metálicos. O comportamento, no entanto, é variado: partindo de uma concentração inicial expressa em percentil, o $\mathrm{Cd}$, o que menos sofreu queda na concentração em solução $(-12,2 \%)$, passou de 100 para 87,8; já o $\mathrm{Pb}$, o mais imobilizado $(-69,1 \%)$, apresentou queda de 100 para 30,9 $(\mathrm{Pb})$ (Tabela 1).

Tabela 1. Fração remanescente de metais nas soluções em diferentes intervalos de tempo de interação solução-solo

\begin{tabular}{lcccccc}
\hline Amostras & & \multicolumn{5}{c}{ Tempo (h) } \\
& & 1 & 3 & 12 & 24 & 48 \\
\hline $\mathrm{Cr}^{3+}(\%)$ & a & 50,0 & 57,5 & 51,5 & 49,2 & 30,0 \\
& b & 38,8 & 32,9 & 18,2 & 15,5 & 10,2 \\
& c & 46,7 & 46,2 & 35,6 & 34,2 & 28,3 \\
$\mathrm{Cu}^{2+}(\%)$ & a & 77,2 & 80,8 & 80,9 & 81,9 & 78,4 \\
& b & 59,8 & 57,8 & 52,3 & 53,9 & 49,4 \\
& c & 78,8 & 77,7 & 75,3 & 76,3 & 76,3 \\
$\mathrm{Cd}^{2+}(\%)$ & a & 78,8 & 88,5 & 91,1 & 88,1 & 88,7 \\
& b & 80,3 & 76,9 & 93,8 & 81,5 & 82,7 \\
$\mathrm{Hg}^{2+}(\%)$ & c & 87,8 & 92,0 & 88,1 & 87,1 & 85,8 \\
& a & 80,9 & 86,4 & 97,2 & 87,3 & 93,8 \\
& b & 65,0 & 59,0 & 76,6 & 58,3 & 43,0 \\
$\mathrm{~Pb}^{2+}(\%)$ & $\mathrm{c}$ & 84,1 & 73,6 & 66,6 & 59,5 & 80,5 \\
& a & 55,1 & 58,9 & 60,9 & 65,7 & 60,9 \\
& b & 30,9 & 29,0 & 24,7 & 25,9 & 24,0 \\
& c & 51,2 & 51,2 & 52,7 & 50,2 & 52,2 \\
\hline
\end{tabular}

Os dados foram obtidos sob condições de pH entre 3,75 e 4,42 e temperatura entre 24,5 e $26,1^{\circ} \mathrm{C}$. a: areia argilosa; b: argila siltosa; c: argila arenosa

A expressão gráfica dos resultados torna evidente a variação da capacidade de retenção nos diferentes grupos amostrais, expressa pela razão entre a quantidade de elementos metálicos retida e a quantidade dissolvida remanescente (Figuras 2 a 4).

A relação entre a concentração de elementos metálicos e os intervalos de tempo de amostragem não é linear, em razão da competitividade entre os elementos ao longo do processo e da flutuação diária de temperatura. De modo geral, é durante o primeiro intervalo amostral que a solução exibe a maior variação na concentração de elementos em solução. Nos intervalos subseqüientes, verifica-se oscilação na trajetória de concentração, sendo registradas variações não significativas do ponto de vista estatístico. A exceção é o cromo, que ampliou de forma constante a redução de sua concentração em solução, atingindo a marca de $83 \%$ de retenção.

As condições descritas acima são condicionadas por processos de precipitação e formação de minerais, provavelmente hidróxidos, e complementadas por sorção finita associada à dispersão de partículas em sistema fechado. O cálculo da capacidade de retenção das argilas e a presença de material em suspensão reforçam esta hipótese. 
Tabela 2. Capacidade especifica máxima de retenção de metais pelo solo durante o experimento

\begin{tabular}{|c|c|c|c|c|c|c|c|c|}
\hline \multirow[t]{2}{*}{$\mathrm{ID}^{1}$} & \multirow{2}{*}{$\begin{array}{c}\text { Classificação } \\
\text { textural }\end{array}$} & \multirow{2}{*}{$\begin{array}{c}\text { Conteúdo de } \\
\operatorname{argila/lama}^{2}(\%)\end{array}$} & \multirow[t]{2}{*}{$\mathrm{CO}(\%)$} & \multicolumn{5}{|c|}{ Retenção (mg/g) } \\
\hline & & & & $\mathrm{Cr}$ & $\mathrm{Cu}$ & $\mathrm{Cd}$ & $\mathrm{Hg}$ & $\mathrm{Pb}$ \\
\hline $\mathrm{a}$ & Areia argilosa & $30,6 / 48,7$ & 1,17 & 1,82 & 0,69 & 0,63 & 0,31 & 4,04 \\
\hline $\mathrm{b}$ & Argila siltosa & $60,7 / 97,7$ & 1,74 & 2,33 & 1,62 & 0,96 & 2,85 & 7,85 \\
\hline $\mathrm{c}$ & Argila arenosa & $54,2 / 58,6$ & 1,27 & 1,86 & 0,72 & 0,80 & 0,98 & 5,53 \\
\hline
\end{tabular}

$\mathrm{CO}=$ carbono orgânico

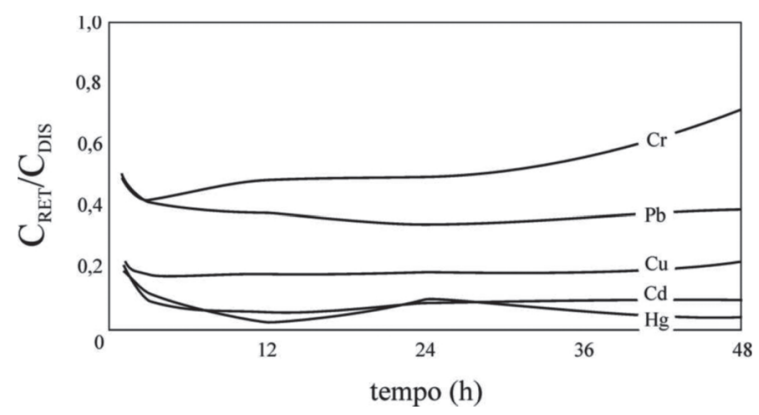

Figura 2. Capacidade de retenção da amostra "a" nos diversos intervalos amostrais, expressa em relação ao conteúdo de metais retido por grama de solo. $C_{R E T}=$ concentração retida e $C_{D I S}=$ concentração dissolvida

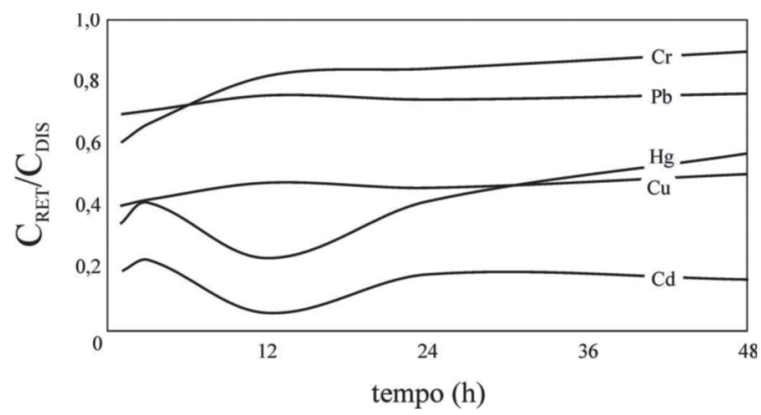

Figura 3. Capacidade de retenção da amostra " $b$ " nos diversos intervalos amostrais, expressa em relação ao conteúdo de metais retido por grama de solo. $C_{R E T}=$ concentração retida e $C_{D I S}=$ concentração dissolvida

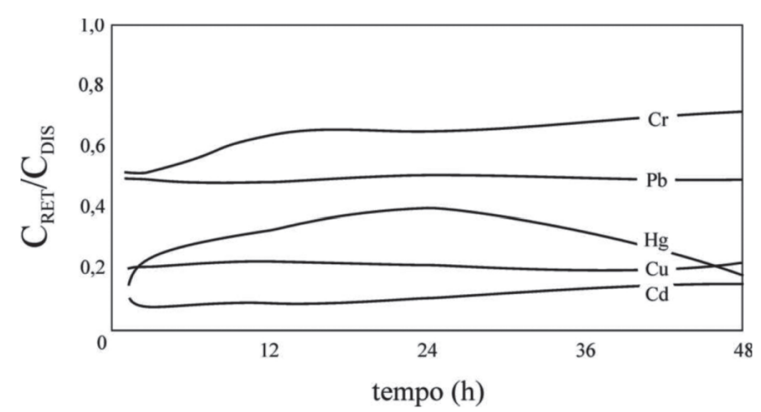

Figura 4. Capacidade de retenção da amostra "c" nos diversos intervalos amostrais, expressa em relação ao conteúdo de metais retido por grama de solo. $C_{R E T}=$ concentração retida e $C_{D I S}=$ concentração dissolvida

Na Tabela 2 encontram-se sumarizados os principais dados resultantes da caracterização dos solos locais.

Os resultados químico-analíticos apontam, para todas as amostras e íons estudados, retenção total na faixa de 7,49 a $15,61 \mathrm{mg} \mathrm{g}^{-1}$. A partir da Tabela 2 fica demonstrado que as amostras com maior teor de carbono orgânico e argilominerais foram as que apresentaram maior retenção. Este fato concorda com os dados teóricos que dão conta de que a capacidade de troca catiônica da matéria orgânica é até 65 vezes maior que a exibida pela caolinita ${ }^{16}$.

Paralelamente, verifica-se que a ordem de seletividade de ele- mentos metálicos não é a mesma nas três amostras de solo: embora $\mathrm{Cr}$ e $\mathrm{Pb}$ sejam os mais retidos, $\mathrm{Cu}, \mathrm{Cd}$ e $\mathrm{Hg}$ trocam de posição no "ranking" obtido a partir do experimento, como apresentado a seguir: $\mathrm{a} \rightarrow \mathrm{Cr}>\mathrm{Pb}>\mathrm{Cu}>\mathrm{Cd}>\mathrm{Hg}$; $\rightarrow \rightarrow \mathrm{Cr}>\mathrm{Pb}>\mathrm{Hg}>\mathrm{Cu}>\mathrm{Cd}$; $\mathrm{c} \rightarrow \mathrm{Cr}>\mathrm{Pb}>\mathrm{Cu}>\mathrm{Hg}>\mathrm{Cd}$.

\section{CONCLUSÃO}

A utilização de testes de retenção em série na avaliação ambiental de áreas industriais demonstrou ser um procedimento rápido que permite determinar a capacidade de retenção dos solos.

$\mathrm{O}$ cromo é, em todas as amostras, o mais retido, fenômeno este associado à maior carga iônica $\left(\mathrm{Cr}^{3+}\right)$. Os demais podem estar submetidos a fenômenos de complexação, precipitação e ao potencial iônico (razão carga/raio) que interferem no equilíbrio dos íons em solução. $\mathrm{O}$ fenômeno de imobilização de elementos depende ainda das características da mineralogia, como conteúdo da fração fina e, também, do conteúdo de matéria orgânica. Após o experimento, pôde-se comprovar que a amostra com conteúdos de argila e matéria orgânica maiores foi aquela que apresentou maior capacidade de retenção de íons metálicos.

Por suas características mineralógicas e orgânicas, os solos locais podem ser utilizados na retenção de metais, tendo capacidade de impedir o transporte e a disseminação através das águas subterrâneas.

\section{AGRADECIMENTOS}

Ao CNPq pelo fomento a pesquisa, concedido através de bolsa de estudos; à REFAP S/A representada por P. J. R. de Freitas, A. R. T. Essinger e J. M. Spier.

\section{REFERÊNCIAS}

1. Krishna, A. K.; Govil, P. K.; Environ. Geology 2005, 47, 1054.

2. Wake, H.; Estuarine, Coastal and Shelf Science 2005, 62, 131.

3. Mysore, D.; Viraraghavan, T.; Jin, Y.; Water Res. 2005, 39, 2643.

4. Petroni, S. L. G.; Pires, M. A. F.; Munita, C. S.; Quim. Nova 2000, 23, 477.

5. Fungaro, D. A.; Silva, M. G.; Quim. Nova 2002, 25, 1081.

6. Bailey, S. E.; Olin, T. J.; Bricka, R. M.; Adrian, D. D.; Water Res. 1999, 33, 2469.

7. Farrah, H.; Hatton, D.; Pickering, W. F.; Chem. Geol. 1980, 28, 55.

8. Yong, R. N.; Mohamed, A. M. O.; Warkentin, B. P.; Principles of contaminant transport in soils, Elsevier: Amsterdam, 1992.

9. Yin, Y.; Lee, S-Z.; You, S-J.; Allen, H. E. Em Environmental restoration of metals-contaminated soils.; Iskandar, I. K., ed.; Lewis Publishers: New York, 2001, p. 77.

10. Roy, W. R.; Krapac, I. G.; Chou, S. F. J.; Griffin, R. A.; United States Environmental Protection Agency Technical Resource Document EPA/530SW-87-006-F, 1992.

11. Fundação de Amparo da Universidade Federal do Rio Grande do Sul (FAURGS), material não publicado.

12. Sanberg, E.; Dissertação de Mestrado. Universidade Federal do Rio Grande do Sul, Brasil, 2003.

13. White, P. A.; Claxton, L. D.; Mutat. Res. 2004, 567, 227.

14. Basílio, M. S.; Friese, K.; Lena, J. C.; Nalini Jr, H. A.; Roeser, H. M. P.; Quim. Nova 2005, 28, 822

15. Shepard, F. P.; J. Sedimentary Petrology 1954, 24, 151.

16. Deutsch, W. J.; Groundwater Geochemistry Fundamentals and Applications to Contamination, $1^{\text {st }}$ ed.; Lewis Publishers: New York, 1997. 